\title{
Characteristics of Economic Growth in Guizhou and its Inspiration to $\mathrm{PM}_{2.5}$ Control
}

\author{
Jizhang Zong ${ }^{1}$, Song $\mathrm{Li}^{1, *}$, and $\mathrm{Yi} \mathrm{Bai}^{1}$ \\ ${ }^{1}$ School of Geography and Resources, Guizhou Education University, Guiyang 550018, China
}

\begin{abstract}
Guizhou province, one of the poorest provinces in China, has attracted the state's close attention. The work selected such data of GDP, per-capita GDP, and industrial structure from Guizhou Statistical Yearbook and Guiyang Statistical Yearbook from 1995 to 2019. Then the spatiotemporal characteristics of county region economy were studied using several methods including spatial autocorrelation (Moran's I), and standard deviation coefficients based on GIS. The results showed that Guiyang and Zunyi were the centers of economic growth in Guizhou. There was a regional differentiation that the growth of county GDP lowered from northwest to southeast. The industrial structure changed significantly in Guizhou's county level in 2005 and 2009, and ITI was 6.7 and 8.3, respectively. There was a noticeable tendency that developed counties had low ITI. In contrast, spatiotemporal characteristics of per-capita GDP growth were more complex, and these counties of high per-capita GDP disbursed over a wide region of the GuiyangZunyi industrial zone. Finally, we analyzed the enlightenment on $\mathrm{PM}_{2.5}$ control in Guizhou.
\end{abstract}

\section{Introduction}

The gap between local regions is harmful to the rapid and stable growth of the country's overall economy and the society's harmonious development[1], and is an urgent problem for balanced regional development of the region[2]. Since the counties were included in the national economic reform category after 2002, as the basis for the healthy and stable development of the national economy[3], it had increasingly been a hotspot[4] in geographical research. Now, the related methods showed a diversified development trend, and the application attention of single index method[5] and comprehensive index method[6] had steadily improved. Socioeconomy had a specific correlation[7] with $\mathrm{PM}_{2.5}$. We took counties of Guizhou as the basic unit and used GIS to analyze the development law and industrial structure change pattern from 1995 to 2019. Consequently, the enlightenment of $\mathrm{PM}_{2.5}$ prevention and control was analyzed.

\section{Methodolgy and data sources}

\subsection{Data sources}

The data sources came from Guizhou Statistical Yearbook and Guiyang Statistical Yearbook. The data of Xiaohe, Zunyi Huichuan, Tongren (Bijiang), and Bijie (Qixingguan) involved in the name change or adjustment of administrative division during the period was finally determined by interpolation and other methods after merging and splitting according to its actual changes.

\subsection{Methodolgy}

The spatial autocorrelation analysis was used to analyze the GDP and per capita GDP of Guizhou from 1995 to 2019, and the spatial change of counties economic development explored. Spatial autocorrelation (Moran's I) calculation formula ${ }^{[7]}$ is as follows:

$$
\begin{gathered}
I=\frac{n \sum_{i} \sum_{j} W_{i j}\left(x_{i}-\bar{x}\right)\left(x_{j}-\bar{x}\right)}{\sum_{i} \sum_{j} W_{i j} \sum_{j}\left(x_{i}-\bar{x}\right)^{2}} \\
E(I)=-1 /(n-1)
\end{gathered}
$$

where $W$ is the spatial weight matrix; $n$ the number of counties; $x_{i}$ the value of county $i$; $\bar{x}$ the average value. The value of Moran's $I$ is $[-1,1]$. When $I$ equals to 0 , each regional unit is independent of each other and spatially uncorrelated, presenting a random distribution. The closer $I$ is to 1 , the stronger the spatial agglomeration; the closer $I$ is to -1 , the stronger the difference from the surrounding area.

ITI (industrial structures translation index) is constructed to measure industrial structure's transfer quantitatively. Generally, the industrial structure had a low-high tendency, and the ITI expression is as follows:

$$
I T I=\sum_{i=1}^{n} \sum_{j=1}^{m} \sqrt{N_{i j}^{2}}
$$

where $i$ and $j$ are the county and industrial structure, respectively; $n$ and $m$ the numbers of counties and industrial structures, respectively; $N$ is the industrial structure's gradient. Table 1 showed the values. Reverse transfer means that the regional economy shifts from the

\footnotetext{
* Corresponding author: zhijinese@163.com
} 
tertiary to the primary industry, with negative ITI; positive transfer means the economy shifts from the primary to the tertiary industry, with positive ITI.

Formula (3) is used to calculate ITI of the previous year and sum it yearly to obtain TITI within a period, namely the total industrial structure transfer index:

$$
\text { TITI }=\sum_{i=1}^{n} \text { ITI }_{i}
$$

where $n$ is the time value of TITI. Here, the number of counties is 88 , and the period is from 1995 to 2019 .

Table1. The gradient value of ITI and TITI

\begin{tabular}{llllllll}
\hline Items & ITI & & & & & \\
\cline { 2 - 7 } & & 123 & 132 & 213 & 231 & 312 & 321 \\
\hline$\exists$ & 123 & 0 & 1 & 2 & 3 & 4 & 5 \\
132 & -1 & 0 & 1 & 2 & 3 & 4 \\
213 & -2 & -1 & 0 & 1 & 2 & 3 \\
231 & -3 & -2 & -1 & 0 & 1 & 2 \\
312 & -4 & -3 & -2 & -1 & 0 & 1 \\
321 & -5 & -4 & -3 & -2 & -1 & 0 \\
\hline
\end{tabular}

\section{Economic evolution and the relations hip with $\mathbf{P M}_{2.5}$ in Guizhou}

\subsection{Temporal evolution characteristics}

The economic structure of Guizhou generally showed a downward trend from northwest to southeast. In contrast, the spatiotemporal development of GDP per capita was more complicated. The growth curves of county GDP per capita and regional GDP in Guizhou showed that both GDP and per capita GDP of counties were in a steady growth trend from 1995 to 2019 . The average annual ITI was 15.4, and $\sigma$ was 8.6 from 1995 to 2019 . The industrial structure changed significantly, with large rangeability.

\subsection{Spatial evolution characteristics}

The Moran's I of per capita GDP was 0.42 , with prominent clustering characteristics. The agglomeration trend fluctuates wildly(Fig.1), and the regional differentiation was significant from 1995 to 2019. The $\sigma$ showed that counties' overall economic disparity gradually decreased. The $\sigma$ of per capita GDP decreased from 1.05 in 2002 to 0.67 . From 1995 to 2019, the main economic cluster of Guizhou was Guiyang-Zunyi industrial belt, followed by Northeast Guizhou and Southeast Guizhou, respectively, showing prominent differentiation characteristics (Fig.1). The results of per capita GDP showed that the areas with high per capita GDP in Guizhou were mainly distributed in the Guiyang-Zunyi industrial belt with the provincial capital Guiyang as the radiation center, and Guiyang and Zunyi as the dual cores, as well as the continuous region of Kaili, Duyun, and Anshun with Guiyang and Zunyi as the cores.

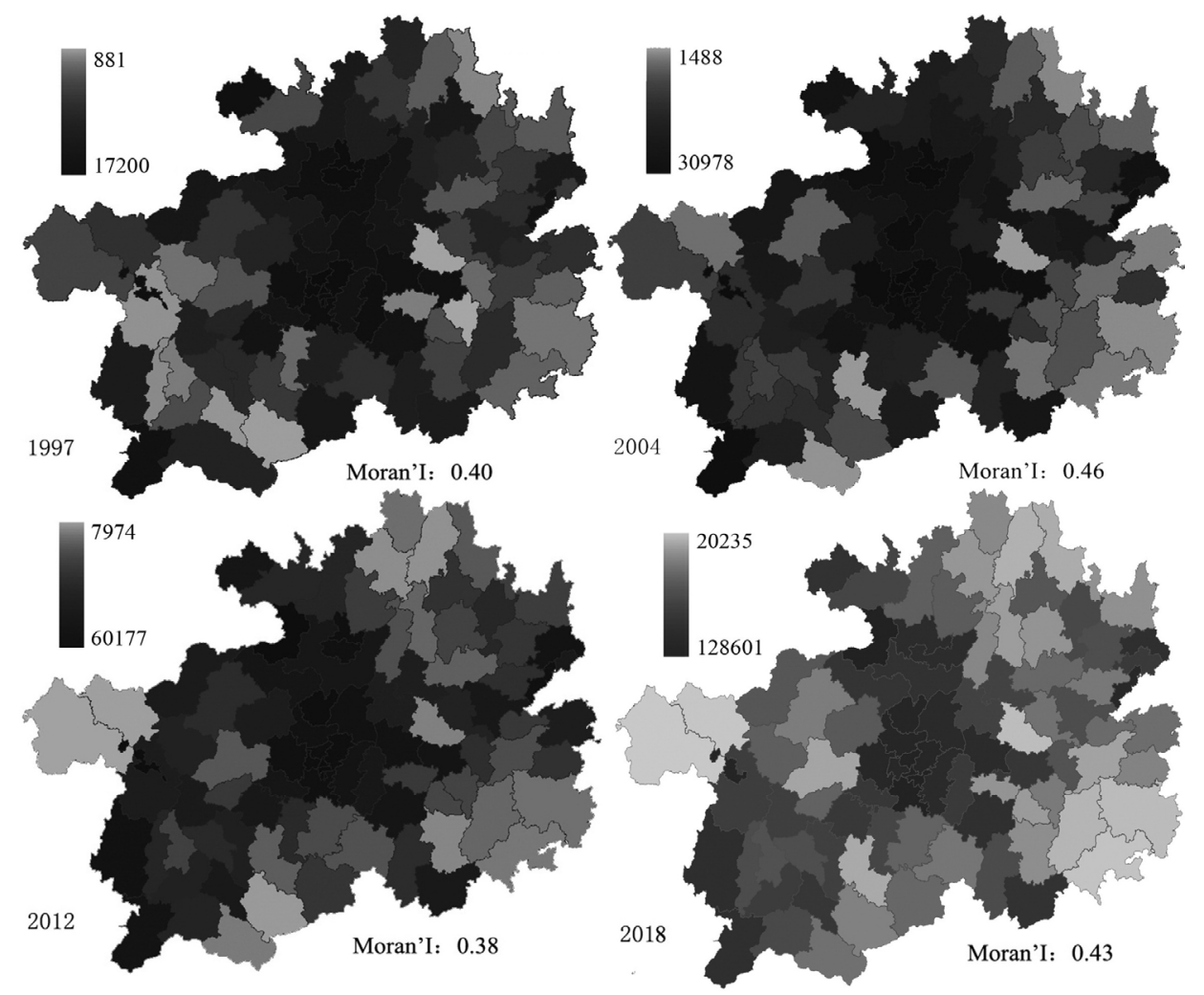

Fig.1 Spatial difference of per-capita GDP and spatial autocorrelation analysis of counties 


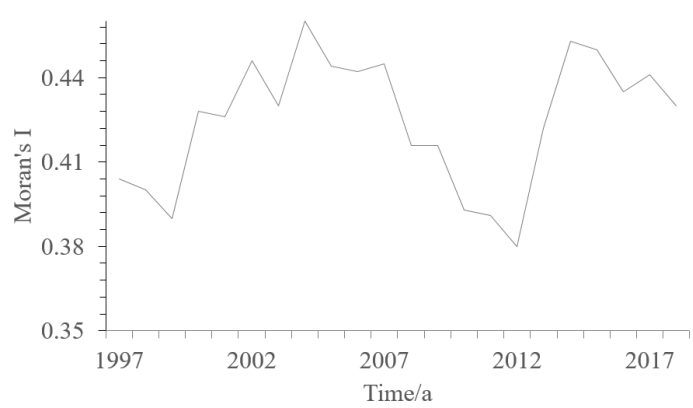

Fig.2 Moran's I of county per-capita GDP in Guizhou

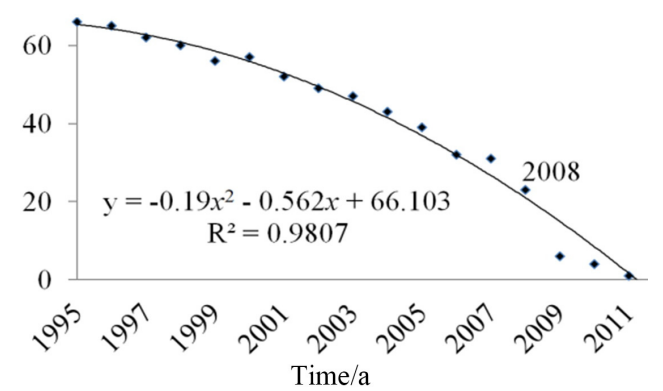

Fig.3 The county dominated by the first industry

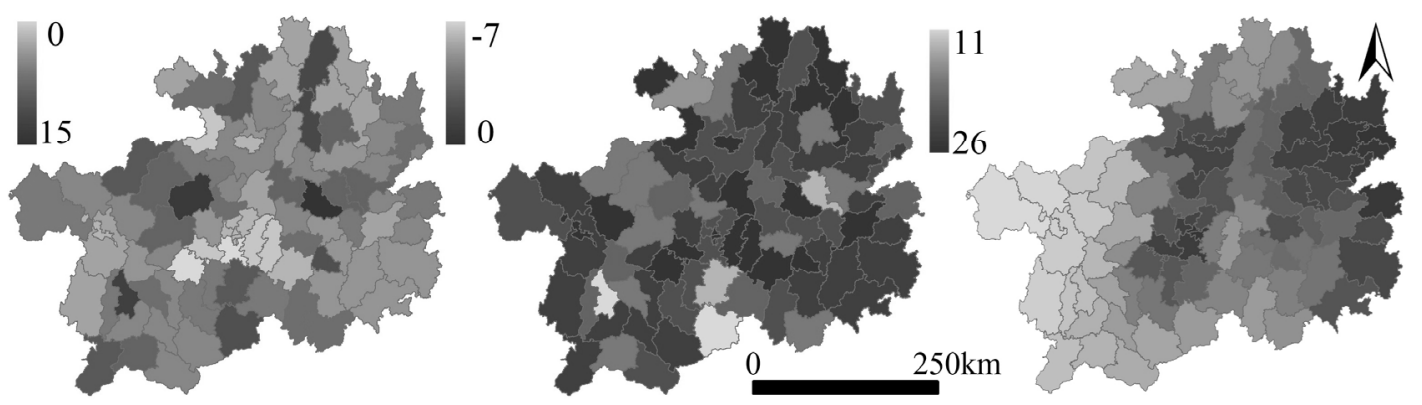

Fig.4 The county TITI(left), ITI(middle) and PM$_{2.5}$ concentration(right,2016) in Guizhou from 1995 to 2019

\subsection{Industrial structure evolution}

Spatial autocorrelation analysis was performed by assign ing attribute 1 to the areas dominated by the primary ind ustry, and attribute 111 to the areas dominated by the oth er. The results showed economic agglomeration effect in Guizhou was weak before 1998, and the Moran's I was s table at about 0.15 . Discrete distribution was carried out for the counties with dominant secondary and tertiary ind ustries, the continuous distribution centered on Guiyang, and the areas, including Xingyi, Panxian, Zhongshan, $\mathrm{Ch}$ ishui, Renhuai, Honghuagang, and Kaili, with dominant secondary and tertiary industries. The Moran's I rose to 0. 39 in 1999, and an axial trend appeared in the counties w here the secondary and tertiary industries dominated (Fig. 2). From 2002 to 2004, Moran's I rose to 0.46, and Guiy ang-Zunyi Belt became a continuous distribution domina ted by the secondary and tertiary industries. In 2007, the Moran's I rose to 0.42 . After 2008, the counties dominate $\mathrm{d}$ by the primary industry had undergone tremendous cha nges, from 23 in 2008 to 6 rocky desertification counties with high mountains and steep slopes such as Weining a nd Yinjiang. It dropped to 4 in 2010, and only Yinjiang $r$ emained after 2011 (Fig. 3).

TITI results were visualized by GIS, and Fig. 4 (left) showed the results. From 1995 to 2019, TITI of counties with a better economic development level was 0 , without changing the industrial structure. TITI of counties with a relatively high economic development level was 1 , and the industrial structure was unchanged. TITI of counties with the highest economic development level was 2 , and there were few industrial structure changes. In counties with relatively backward economic development, $T I T I \geq 13$, and industrial structure changed frequently.

The unchanged ITI was set to 0 , and the changed set to negative values (Fig.4(right)). Generally, reverse ITI showed a similar spatial distribution pattern to TITI.
Except Yinjiang was relatively economically backward, Pan County belonged to resource-based development pattern, and other counties were all provincial capitals and prefecture-level capital cities, which belonged to relatively developed counties without reverse changes. Counties with $I T I \leq 2$ and reverse changes in the industrial structure had a higher economic development. Counties with $I T I \geq 5$ and reverse changes in the industrial structure, were mostly remote areas with a relatively backward economy.

\subsection{Relationship between $\mathrm{PM}_{2.5}$ and economy}

We used the global annual average raster data of $\mathrm{PM}_{2.5}$ published by Columbia University International GeoInformation Science Information Network Center. The data from 1998 to 2016 were based on GWR methods, with satellite remote sensing for aerosol optical depth retrieval. ArcGIS were used in the counties of Guizhou to obtain $\mathrm{PM}_{2.5}$ data in 2016. The obtained annual average value of $\mathrm{PM}_{2.5}$ was compared with economic data to find the correlation between economic activities and $\mathrm{PM}_{2.5}$. The direct analysis showed among the GDP, the three industries, and $\mathrm{PM}_{2.5}$, the correlation coefficient between $\mathrm{PM}_{2.5}$ and the primary industry was -0.57 , and that between $\mathrm{PM}_{2.5}$ and the registered population was slightly less than -0.5 . It was related to the significant improvement in air quality in 2016 and the backward socio-economic level of Guizhou.

\section{Mechanism and inspiration to $\mathrm{PM}_{2.5}$ control}

\subsection{Natural Environment}

There were two cases in Guizhou where the industrial structure was stable. One was relatively high economic 
development and a stable and mature industrial structure. This case referred to the areas with the lowest topographical undulations distributed in central Guizhou. The other kept a low economic development level and lacks the areas with optimized industrial structure. This case was in northern and southeastern Guizhou, where the latter was the largest distribution area of karst. Topography was another significant impact on economic development. The county per capita GDP had a significant negative correlation with the average relative height difference, with a correlation coefficient of -0.5 .

\subsection{Location and resources}

Resource endowments affected the regional economy and the optimization of industrial structure. In Guiyang, the tertiary industries of Yunyan and Nanming were dominant, while Wudang, Baiyun, and Huaxi all took advantage of the secondary industries. Duyun, Kaili, Zunyi, and Anshun had a relatively high development level due to their advantageous locations. Their tertiary industries were dominant, while the proportions of primary industries were significantly lower. The industrial structures of Liuzhi, Panxian, and Shuicheng were directly related to coal resources. In the rocky desertification areas such as Leishan and Majiang, the harsh geographical environment and lack of resources have severely restricted the primary industry's development. Even a slight improvement in regional conditions, the proportion of the tertiary industry had risen significantly. Chishui and Renhuai also took advantage of the secondary industry. The advantages of Chishui's secondary industry were mainly due to its geographical advantage at the junction of the three provinces of Guizhou, Chongqing and Sichuan, and the unique tourism resources. At the same time, Renhuai had a relatively high level of economic development due to the influence of the Maotai Group.

\subsection{Labour force}

The permanent population dropped from 39.04 million in 2004 to 36.23 million in 2019 , a decrease of nearly 2.81 million. The number of the labor force, comprehensive quality, and consumption level affected the regional economy and the industrial structures. In 1997, the labor force ratio of primary, secondary and tertiary industries was 7:1.5:1.5, respectively. Among urban labor forces, college graduates and technical school graduates accounted for only $12.5 \%$. In 2019 , the labor force ratio of primary, secondary and tertiary industries was 5.3:1.8:2.9, respectively. Among the urban labor force, college graduates and technical school graduates accounted for only $23.46 \%$, the quality of the labor force has improved significantly. The increased proportions of the labor force in the secondary and tertiary industries and their quality improvement have directly affected the development of the secondary and tertiary industries, and leading to the adjusted industrial structure and the developed regional economy.

\subsection{Inspiration of $\mathrm{PM}_{2.5}$ control}

$\mathrm{PM}_{2.5}$ control should comprehensively consider natural and social-economic factors, including the natural environment, location, resource endowments, and population (such as labor force). Statistics showed that the county-level administrative districts with the highest average $\mathrm{PM}_{2.5}$ concentration in Guizhou Province in 2016 included Yunyan District, Nanming District, Bijiang District, Wanshan District, Huaxi Guanshanhu District. Except for the resource-based city Wanshan, other cities were the capitals of Guiyang City and prefecture-level administrative regions. Weining, Hezhang, Nayong, Qinglong, and Pu'an had the annual lowest-average concentrations. These counties kept relatively low economy in relatively remote regions.

\section{Conclusion}

Guizhou $\mathrm{PM}_{2.5}$ was restricted by geographical location, resources, and natural environment. When analyzing the regional economic development level, only by comprehensively using average indicators and total indicators could we draw more realistic research conclusions. This problem could be pronounced in karst provinces such as Guizhou. Due to the fragile ecological environment and broken karst landforms, Guizhou has minimal conditions for developing primary industries. With less resource endowments, some remote rocky desertification mountainous areas have a low economic aggregate but a high proportion of the tertiary industry. Therefore, GIS technology and economic geography theory were used to analyze economic and $\mathrm{PM}_{2.5}$ disaster issues, which objectively and scientifically reflected the temporal and spatial patterns of regional development and regional development laws. It can provide a valuable reference for regional development.

\section{Acknowledgments}

This work was funded by the project of the National Natural Science Foundation of China(41861035), the project of Guizhou Province Education Department for Tip-top Talent of Science and Technology(Qianjiaohe KY[2016] 082), and the project of Guizhou Province Science and Tecnology Agency(Qiankehe Zhicheng[2018] 2776, Qiankehe Jichu[2016] 1112, Qiankehe Jichu[2018] 1120).

\section{References}

1. Yang Zhibin, Zeng Xianfeng. A Summary of the Research on china's regional economic disparities. Economic Geography, 30(6): 899-905(2010)

2. Gardiner B, Martin R, Sunley P, et al. Spatially unbalanced growth in the British economy. Journal of Economic Geography, 13(6):889-928(2013)

3. Wang Xichao, Cui Aiping, Jin Hao. Application of factor analysis in the evaluation of county economic 
development level. Journal of Hebei University of Economics and Business, 32(6): 90-96(2011)

4. Du Ting, Xie Xianjian, Liang Haiyan, et al. Comprehensive evaluation and spatial analysis of county economy in Chongqing based on entropy weight TOPSIS and GIS. Economic Geography, 34(6): 40-47(2014)

5. Wang Yanhui, Li Jingyi. Evaluation of coupling and coordination between ecological environment quality and economic development level in contiguous destitute areas. Chinese Journal of Applied Ecology, 26(5): 1519-1530(2015)

6. Zhao Xuyang, Liu Zheng, Zhao Haijian. Research on the coupling mechanism of economic development and ecological environment in mountainous areas: Taking Pingshan County of Hebei Province as an example. Research of Soil and Water Conservation, 21(3): 176-181(2014)

7. Yang Kun, Yang Yulian, Zhu Yanhui, Li Cen, Meng Chao. Spatial relationship and causes of China's $\mathrm{PM}_{2.5}$ pollution and social economy. Geographical Research, 35(06): 1051-1060(2016) 